\title{
Astronomical interferometry,
}

\section{from the visible to sub-mm waves}

\author{
Andreas Quirrenbach \\ University of California, San Diego
}

\begin{abstract}
The history of astronomical interferometry dates back to the $19^{\text {th }}$ century, when Fizeau, Stéphane and later Michelson set out to measure the angular diameters of stars. Whereas Stéphane could only obtain an upper limit of 0.158 arcseconds for the stars he observed in 1874, Michelson published interferometric diameters of Jupiter's Galilean moons in 1891. These early experiments used masks with two small openings placed on the aperture of astronomical telescopes to perform double-slit experiments à la Young. Around 1920 Michelson increased the resolution by placing a periscope on the front-end of the 100 inch telescope on Mt. Wilson; this arrangement enabled him and his collaborators to determine the orbit of the binary system Capella, and to measure the diameter of Betelgeuse. After these initial successes, technological difficulties slowed further progress for several decades. In the 1950's and 60's Hanbury Brown, Twiss, and collaborators invented intensity interferometry ${ }^{1}$ and used it to measure the diameters of 32 stars - this is still the best data set on hot stars available today.

The era of modern stellar interferometry began in 1974 when Labeyrie successfully combined the light from two telescopes spaced by $12 \mathrm{~m}$. This breakthrough was soon followed by the construction of similar instruments, which took advantage of modern electronic detectors and computer control to track and stabilize the interference fringes with real-time servo loops, which drive optical delay lines that compensate for the optical pathlength difference and thus maintain coherence between the two arms of the interferometer (see Figure 1). This active pathlength control with millisecond time resolution is a crucial aspect of ground-based astronomical interferometry, because the Earth's rotation and random atmospheric fluctuations have to be tracked continuously.
\end{abstract}

\section{Interferometry and stellar astronomy}

With an angular resolution approaching $\lambda / B \approx 500 \mathrm{~nm} / 100 \mathrm{~m} \approx$ 1 milliarcsecond, optical and near-IR interferometers have opened completely new avenues for stellar astronomy. A few hundred stellar diameters have been measured with $\sim 1 \%$ precision; these data are of fundamental importance for the determination of stellar surface temperatures. The masses of about two dozen stars in binary systems have been derived from the application of Kepler's Laws to their orbits measured by a combination of interferometric and spectroscopic radial-velocity data. Interferometry has been used to infer the distance of a nova explosion, and of Galactic Cepheid stars. These are important measurements because they demonstrate the potential of interfer-ometric techniques to contribute to cosmology: Cepheid distances are the first rung on the cosmic distance ladder, which defines the size and through the Hubble constant - the age of the universe. Closer to home, interferometric diameters of cool Mira variables hold important clues to the mechanism that makes them pulsate with very large amplitudes.
New experimental techniques usually bring surprises, and optical interferometry is no exception to that rule. When we used the Mark III Interferometer to observe cool giant stars, we found that their diameters vary with wavelength. This effect can in principle be understood by considering the effect of TiO molecules in the stellar photosphere, which blocks the view of lower layers at wavelengths corresponding to the molecular absorption bands. So one sees a higher layer - corresponding to a larger radius inside TiO bands than in the continuum between the bands. However, we had not expected that the difference would be so large that we could easily measure it! More recently, a near-IR interferometer in Arizona was used to observe a class of stars technically known as Herbig Ae/Be stars. These are young stars with roughly 2 to 5 solar masses, which still show indications of the presence of circumstellar gas and dust - the material from which the star formed in the first place. According to the standard paradigm this circumstellar material should be organized in a disk, and such disks had previously been observed with millimeter interferometry around a number of these stars. But the near-IR interferometer observations indicate that the matter around Herbig Ae/Be stars is distributed in a spherically symmetric way, in apparent contradiction to the millimeter-wave results. A possible solution to this dilemma involves a more complicated structure, in which spherically distributed material is surrounded by a much larger disk. Only future observations will tell whether this is the correct answer.

\section{Imaging interferometry}

The results described so far have been obtained with simple "two-element" interferometers. The information that can be obtained with such instruments is quite limited, however; in essence they measure only one Fourier component of the brightness distribution $I(\xi, \eta)$ on the sky at a time:

$$
\text { (1) } \Gamma(u, v)=V e^{i \varphi}=\iint I(\xi, \eta) e^{-2 \pi i(u \xi+v \eta)} d \xi d \eta \text {, }
$$

where $(u, v)$ is the vector connecting the two telescopes, $V$ and $\varphi$ are the amplitude and phase of the measured fringes, and $\xi$ and $\varphi$ are the coordinates in the tangent plane of the sky. To make things worse, the phase of this Fourier component is normally corrupted by atmospheric turbulence and thus rendered useless. During the past few years, however, several multi-telescope arrays have been constructed, which can obtain many Fourier components at a time, $n(n-1) / 2$ for an $n$-element array. Furthermore, such arrays can preserve some phase information; taken together these two improvements over single-baseline interferometers allow true imaging of objects whose structure is not known a priori, by inverting Equation (1) through an inverse Fourier transform. So far, only simple objects such as binary stars have been imaged with separate-element interferometers, but experiments with a multi-aperture mask on the 10-m Keck II telescope 


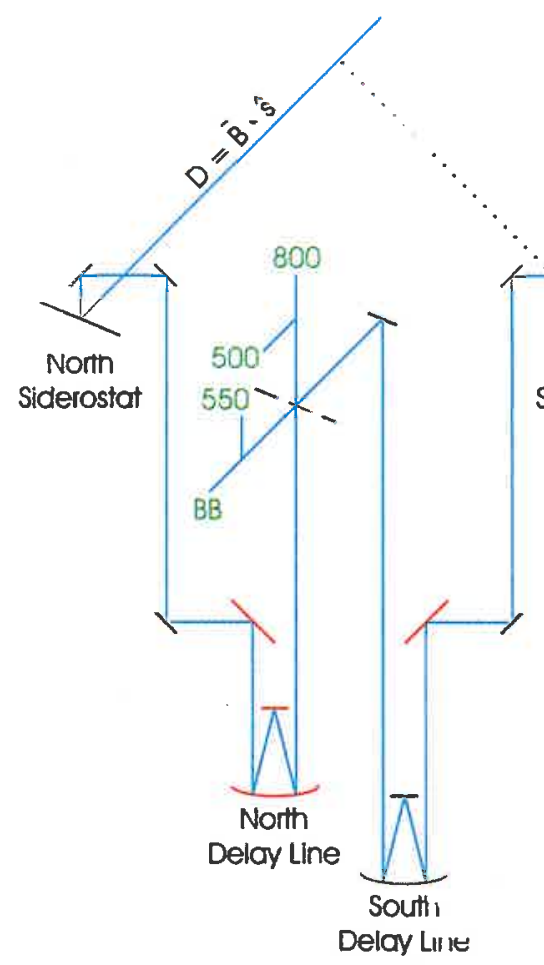

Fig. 1: Schematic drawing of the Mark III Interferometer, a typical two-element stellar interferometer constructed in the 1980 's and operational on Mt. Wilson, CA, until 1992. Light collected with the siderostats is fed into the optical delay lines with two (plane) mirrors mounted on piezo-electric actuators (red), which are part of a servo loop that keeps the two images of the star aligned with each other. The positions of the two optical delay lines are continuously monitored with a laser interferometer. They are optically equivalent, but the cart and the small mirror in one of them (red) are actively controlled and are part of the fringe-tracking servo loop. The beams from the two arms are combined with a $50 \%$ reflective mirror. The light in each of the two outputs is divided with a dichroic beam splitter, so that four wavelength channels (broad band for fringe tracking, $500 \mathrm{~nm}, 550 \mathrm{~nm}$, and $800 \mathrm{~nm}$ ) are available simultaneously. The small mirror in the North delay line is dithered back and forth by one wavelength with a triangle wave at a rate of $500 \mathrm{~Hz}$, so that temporal fringes (alternating constructive and destructive interference) can be detected with photo multiplier tubes in the four channels. The phase of the signal in the broadband channel provides the error signal for the fringe-tracking servo. The useful aperture of the Mark III was $5 \mathrm{~cm}$ (!), which resulted in a limiting magnitude of 5 , i.e., only stars visible with the naked eye could be observed.

have yielded beautiful images of a spiral dust pattern in a binary star system. (Back to the roots and to Fizeau's and Michelson's original masking technique!)

Whereas many interesting observing programs involve bright stars, there is also a strong desire to push interferometry to fainter limiting magnitudes, which means that larger telescopes have to be used. Several projects are now underway to turn some of the world's largest telescopes into interferometer arrays. Most prominent among these are the Keck and VLT Interferometer projects, which both achieved the milestone of "first fringes" in the spring of 2001 (see Figure 2). These facilities will broaden the scope of optical and infrared interferometry by providing sufficient sensitivity to observe rather faint stars and even some extragalactic objects, by offering a suite of beam combiners and detectors covering a wide wavelength range, and by making a fairly complicated technique more accessible to a broad users' community.

\section{Astrometry based on interferometry}

In addition to simple parametric measurements and imaging, interferometry can be used for very precise astrometry, i.e., for the determination of positions and motions of stars. The principle of interferometric astrometry can easily be understood from Figure 1. Because a stellar interferometer (unlike a laser interferometer in the lab) works with a very wide bandwidth, typically $\delta \lambda \geq 0.2 \lambda$, the coherence length is small, and there is a welldefined central fringe in the fringe packet. When the fringe sensor locks on this central fringe, we know that the total pathlength is zero, which means that the internal pathlength difference given by the delay line positions is equal to the external pathlength difference $D=\vec{B} \cdot \hat{s}$. Since the delay line position is continually measured with an internal laser interferometer, we know $D$ and thus the angle between the baseline vector and the direction to the star; this is a basic astrometric datum. It is possi-

ble to perform such delay measurements with a precision of $\sim 10$ $\mathrm{nm}$; for a $100 \mathrm{~m}$ baseline this results in a precision of $\sim 0.1$ nanoradian or 20 micro-arc-seconds. This precision would have enabled Mission Control in Houston to watch Neil Armstrong wiggle his index finger upon leaving the Eagle - without any need for taking a camera along on the ride to the Moon.

Interferometric astrometry has attracted much interest because of its potential to detect extrasolar planets through the reflex motion of their parent stars. This technique is simi-

This precision would have enabled Mission Control in Houston to watch Neil Armstrong wiggle his index finger upon leaving the Eagle

with a $10 \mathrm{~m}$ baseline scheduled for launch in 2009 - will push the astrometric precision down to $\sim 1$ microarcsecond from a vantage point above the Earth's atmosphere, sufficient to detect Earth-like planets around a few nearby stars. ${ }^{2}$ A subsequent space interferometer operating at mid-infrared wavelengths $(\sim 7$ $-20 \mu \mathrm{m})$ may then obtain direct images resolving these planets from their parent stars, and perform low-resolution spectroscopy of their atmospheres. In our wildest dreams, we may hope to discover absorption bands of ozone, signaling the presence of 


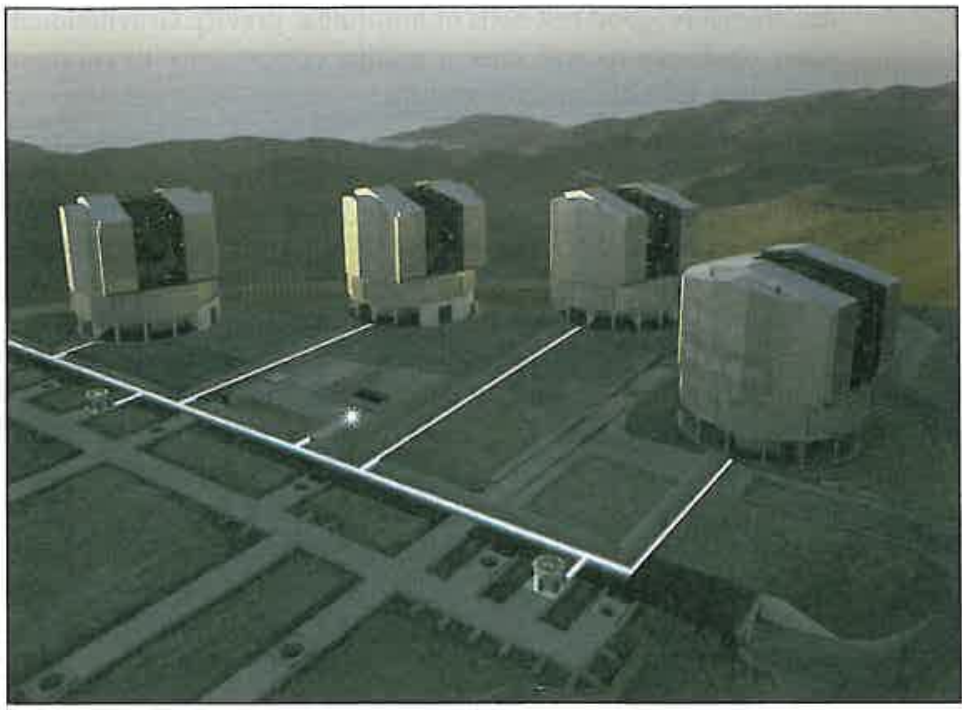

Fig. 2: The Very Large Telescope (VLT) array operated by the European Southern Observatory on top of Cerro Paranal, Chile. This photo shows the four $8.2-\mathrm{m}$ Unit Telescopes (UTs) and various installations for the VLT Interferometer (VLTI). Three movable 1.8-m VLTI Auxiliary Telescopes (ATs), which will be installed in the near future, and paths of the light beams have been superposed on the photo. Also seen are some of the 30 stations where the ATs will be positioned for observations and from where the light beams from the telescopes can enter the interferometric tunnel below. The straight structures are supports for the rails on which the telescopes can move from one station to another. The interferometric laboratory (partly subterranean) is at the center of the platform. The VLTI saw "first fringes" in March 2001. It will be a versatile facility for observations of stars, circumstellar matter, and active galactic nuclei. (Photo courtesy of European Southern Observatory)

oxygen released into the atmosphere by living organisms not too dissimilar from those inhabiting the Earth.

\section{Sub-mm and $\mathrm{mm}$-wave interferometry - the next big leap}

Radio astronomers realized in the 1950's that they can take advantage of Equation (1) and build imaging interferometers much more easily than their colleagues working in the visible wavelength region. After all, they have to maintain coherence only on the $\mathrm{cm}$ (and not sub- $\mu \mathrm{m}$ ) level, and they have to put up with atmospheric fluctuations only on a timescale of minutes, not milliseconds. Radio synthesis imaging has thus become a thriving field, with ever-increasing baseline lengths (now exceeding the diameter of the Earth in ground-space very long baseline interferometry), and at increasingly higher frequencies. There are now a number of $\mathrm{mm}$-wave interferometers that perform imaging of continuum and line emission of objects as varied as nearby star-forming regions and distant galaxies in the young universe, with a spatial resolution of about 1 arc second. These instruments are now beginning to face some of the same difficulties that plague optical and infrared interferometry: fast phase fluctuations induced by atmospheric turbulence, the need to build antennae with smooth surfaces and small thermal and gravitational flexure for use at high frequencies, the large collecting areas required for observations of faint objects, and the limited transparency of the atmosphere in the infrared - sub-mm - mmwave region.

The next big leap in sub-mm and mm-wave interferometry will therefore require a truly global cooperation. Europe, Japan, North America, and Chile have entered a collaboration to construct the Atacama Large Millimeter Array (ALMA) at a site located at an elevation of $5,000 \mathrm{~m}$ above sea level in the Chilean Andes. At this elevation and in an extremely dry climate, the atmosphere is transparent in many frequency bands that are inaccessible from other sites. ALMA will consist of sixty-four 12$\mathrm{m}$ antennas distributed over an area $14 \mathrm{~km}$ in diameter and cover the wavelength range from $0.3-10 \mathrm{~mm}$ (corresponding to frequencies from $30 \mathrm{GHz}-1 \mathrm{THz}$ ). ALMA will be complementary to large optical telescopes by giving us a view of cold objects and those regions of the universe that are hidden from our view by large amounts of dust. These are mostly regions of active star formation, both in our own Galactic backyard, and in nascent galaxies giving birth to the first stars during the dawn of the universe. The development of interferometry, from the visible to sub-mm waves, will thus have a profound impact on our view of our place in the cosmic chain of events leading from the formation of the first galaxies out of fluctuations created in the aftermath of the Big Bang to the evolution of life on our home planet.

' An intensity interferometer compares the intensities, not the amplitudes, at two points in the wavefront. It is interesting to note that this technique, invented for measurements on astronomical scales, is now widely applied to experiments of sub-microscopic objects. It is possible, for example, to determine the size of the "fireball" in heavy-ion collisions with the Hanbury Brown-Twiss method from correlations between the emitted hadrons.

${ }^{2}$ One may ask how an instrument with a resolution of $\sim 10$ milli-arc-seconds may perform measurements with $\sim 1$ microarcsecond precision.

Note, however, that the centroiding accuracy in any measurement is given by the resolution divided by the signal-to-noise ratio. The trick is to keep systematics at a level that enables photon noise-limited observations with an SNR of 10,000 .

\section{About the author}

Andreas Quirrenbach is a Professor of Physics and member of the Center for Astrophysics and Space Sciences at the University of California, San Diego. He became fascinated with the concept of milli-arc-seconds as an undergraduate student while attending a summer school at the Max-Planck-Institut für Radioastronomie (MPIfR). After completing his thesis at MPIfR and obtaining a PhD in astronomy from the University of Bonn, he decided to do "fringe science" at shorter wavelengths and joined the optical interferometry group at the Naval Research Laboratory in Washington, DC, which gave him the opportunity to spend 200 nights on Mt. Wilson observing with the Mark III Interferometer. Subsequently he became a staff member at the Max-Planck-Institut für Extraterrestrische Physik in Garching, where he helped shape the scientific program and instrument complement of the VLT Interferometer. He is now Data Scientist and Member of the Science Team for the Space Interferometry Mission. 